found to have a positive TTG (5\% of cohort, $6 \%$ of those tested). CD was confirmed by endoscopic biopsy in three patients, with two not undergoing endoscopy locally due to their geographic location, giving a prevalence of $\mathrm{CD}$ in this cohort of $5-7 \%$.

The most common site of fracture was the metatarsals $(5 / 7$, $71 \%$ ), with hallux sesamoid and fibula fractures detected in one patient each respectively. Six patients with possible or confirmed CD underwent DEXA. No patients had osteoporosis, with osteopenia (T-Score between -1.0 and -2.5) found in three patients $(3 / 6,50 \%)$. Coexistent vitamin and mineral deficiency were common in the CD patients (iron, 2/6 (33\%); vitamin B12, 2/5 (40\%); folate, 1/4 (25\%); vitamin D, 2/6 (33\%)).

Conclusions In this cohort of patients with stress fractures, the prevalence of $\mathrm{CD}$ was between $5 \%$ and $7 \%$, approximately 5 fold higher than general UK population estimates. Screening for CD with serological testing should be considered in all patients presenting with stress fractures. Stress fractures were not associated with osteoporosis in the patients with CD, and only $50 \%$ had osteopenia, suggesting that coeliac disease and the associated malabsorption leads to stress fracture via mechanisms separate from reduced bone density.

\section{PWE-023 COELIAC DISEASE - OLDER PATIENTS HAVE THE MOST EXTENSIVE SMALL BOWEL INVOLVEMENT ON CAPSULE ENDOSCOPY}

Stefania Chetcuti Zammit* ${ }^{*}$ David S Sanders, Reena Sidhu. Sheffield Teaching Hospitals, Sheffield, UK

\subsection{6/gutjnl-2019-BSGAbstracts.311}

Introduction The relationship between symptomatology, serology and findings on small bowel capsule endoscopy (SBCE) in patients with coeliac disease (CD) remains unclear. Clarifying such associations will help determine if symptoms and serology can predict severity and extent of disease on SBCE.

Methods Patients with newly diagnosed CD (villous atrophy on duodenal histology and positive $\mathrm{CD}$ serology) were recruited. Patients underwent a SBCE at the time of diagnosis. Information on SBCE was recorded. Signs and symptoms at presentation, serological markers, histological classification of disease in the duodenum were noted.

Results Sixty patients with newly diagnosed CD (mean age 44.9 years $\mathrm{SD} \pm 17.4,17$ - 76) were included in this study. Older patients $(p=0.025)$ and patients presenting with iron deficiency anaemia had more extensive small bowel (SB) involvement $(p=0.026)$. Patients presenting with weight loss were more likely to have SB involvement beyond the duodenum $(\mathrm{p}=0.027)$. Patients presenting with iron deficiency anaemia $(p=0.038)$ and weight loss $(p=0.009)$ were significantly older at diagnosis. Serum albumin was lower in those patients diagnosed later on in life $(\mathrm{p}=0.007)$.

There was no significant association between anti-tissue transglutaminase antibody $(p=0.396)$ and extent of affected SB mucosa.

Patients with more severe Marsh classification of disease on histology from the duodenal bulb had more extensive SB involvement $(p=0.017)$.

Conclusions This is the largest study on newly diagnosed CD and SBCE. Older patients are likely to have more extensive disease on SBCE at diagnosis. Symptoms and serology had no impact on the findings on SBCE apart from weight loss and iron deficiency anaemia.

\section{REFERENCE}

1. Lau M, Mooney P, White W, et al. PWE-058 The Role of A Point of Care Test, Simtomax, in Predicting Histological Remission in Coeliac Disease on A Gluten Free Diet. Gut 2016;65:A166-A167.

\section{PWE-025 PANORAMIC VERSUS AXIAL SMALL BOWEL CAPSULE ENDOSCOPY IN OVERT OBSCURE GASTROINTESTINAL BLEEDING}

Stefania Chetcuti Zammit ${ }^{\star}$, Mark E McAlindon, Reena Sidhu. Sheffield Teaching Hospitals, Sheffield, UK

\subsection{6/gutjnl-2019-BSGAbstracts.312}

Introduction Literature comparing axial small bowel capsule endoscopy (SBCE) (Pillcam, Given Imaging) and panoramic SBCE (Capsocam, Capsovision) in obscure gastrointestinal bleeding (OGIB) is limited and contradictory [1,2].

Methods Consecutive patients who presented with overt OGIB at a tertiary centre over a 5 year period underwent either Capsocam SBCE or Pillcam SB3 SBCE. All had negative gastroscopies and colonoscopies/CT colonographies. SBCEs were reviewed by 2 experts. Findings in the 2 groups were compared.

Results 94 patients (39.4\% Capsocam; 60.6\% Pillcam; 57.4\% males; mean age $64.3 \pm 18.0$ years) were included. Both groups were age $(p=0.174)$ and gender $(p=0.137)$ matched. Severity of anaemia $(p=0.053)$ and duration of anaemia $(p=0.264)$ were similar in both groups.

There was no difference between groups in incomplete SBCEs $(\mathrm{p}=0.151)$.

Diagnostic yield (DY) was comparable in both groups but Pillcam had a higher DY than Capsocam in the stomach (table $1)$.

\begin{tabular}{|c|c|c|c|}
\hline & Capsocam n(\%) & Pillcam n(\%) & Significance (p) \\
\hline Gastric DY & $3(8.1)$ & $15(26.3)$ & 0.033 \\
\hline Small bowel (SB) DY & $17(45.9)$ & $25(43.9)$ & 0.842 \\
\hline Colon DY & $2(5.4)$ & $3(5.3)$ & 0.976 \\
\hline
\end{tabular}

Capsocam identified blood (2, 5.4\%), erosions (1, 2.7\%) and ulcers $(1,2.7 \%)$ in the stomach. Pillcam showed blood (4, 5.4\%), erosions $(11,19.3 \%)$, ulcers $(2,3.5 \%)$, varices (1, $1.8 \%)$ and GAVE $(1,1.8 \%)$ in the stomach.

Patients who underwent Capsocam examination had the following findings in the SB: 1 (2.7\%) ulcer, $7(18.9 \%)$ angioectasia, 8 (21.6\%) blood, $2(5.4 \%)$ erosions, 1 (2.7\%) tumour, 1 (2.7\%) diverticulum. Patients who underwent Pillcam had these findings in the SB - 10 (17.5\%) ulcers, 11 (19.2\%) angioectasia, 7 (12.3\%) blood, 19 (33.3\%) erosions, 1 (1.75\%) tumour, 2 (3.51\%) diverticulum, 2 (3.51\%) phlebactesia, $4(7.02 \%)$ polyps, $1(1.75 \%)$ haemangioma, 1 $(1.75 \%)$ intussusception.

Conclusions SB DY was comparable between Capsocam and Pillcam groups in patients with overt OGIB but Pillcam offered a better gastric DY than Capsocam. This might be of 